\title{
Nanosphere lithography with variable deposition angle for the production of one-directional transparent conductors.
}

\author{
Valerio Oddone ${ }^{*}, 1$ and Michael Giersig ${ }^{1}$ \\ ${ }^{1}$ Department of Physics, Freie Universität Berlin, Arnimallee 14, 14195 Berlin, Germany
}

Keywords Transparent electrode, nanosphere lithography, physical vapour deposition, nanolithography

*Corresponding author: e-mail valerio.oddone@gmail.com, Phone: +49 83854294

\begin{abstract}
The production of high quality and cheap transparent electrodes is a fundamental step for a variety of optoelectronic devices. We present a method for the production of transparent conducting films optimised for electrical conduction in one direction. The deposition of a metal film through a perfectly aligned nanosphere-lithography mask at variable incidence angle gave origin to parallel nanowires with thin interconnections. This structure showed excellent conductivity in one direction and high optical transparency.
\end{abstract}

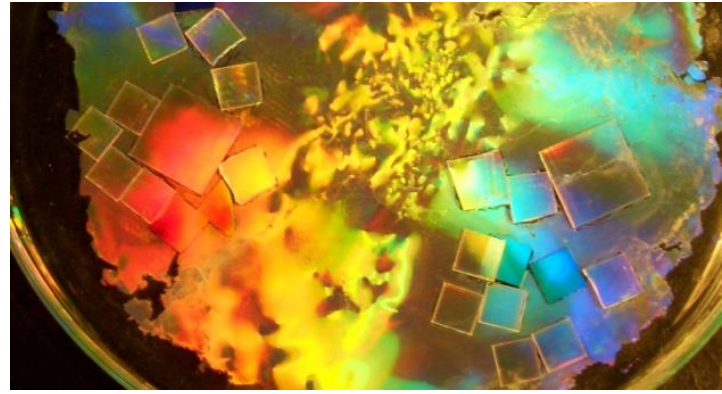

Glass substrates under the crystalline areas of the polystyrene-nanospheres mask
1 Introduction Optoelectronic devices such as OLEDs, flat displays, touch screens and photovoltaic cells usually need a transparent conductor (TC) as top electrode. Moreover, transparent conductors are used for frost-free windows, antistatic layers, transparent antennas and electromagnetic shields [1]. Commonly, tin-doped indium oxide, or ITO [2], is used. ITO has high transparency in the visible and near ultraviolet, and a high charge-carrier density providing sufficient electrical conductivity for most applications [3]. Disadvantages of ITO are the scarcity and costs of indium [4], the low mechanical flexibility [5], the degradation over time and its work function and roughness limiting the hole-injection efficiency [6,7] in semiconductors. Other transparent conducting oxides such as aluminium doped zinc oxide [8,9], fluorine doped tin oxide [10] as well as graphene, carbon nanotubes [11] and organic polymers like PEDOT:PSS [12] are alternative TC under investigation.

If wire grids are used as TC for semiconducting devices, the spacing between the wires must be lower than the diffusion length of the minority charge carriers [13,14], which ranges from 10-20 nm for organic solar cells [15], approximately $300 \mathrm{~nm}$ for amorphous silicon [16] and over $100 \mu \mathrm{m}$ for thin-film crystalline silicon solar cells [17].
Several approaches were used for the production of nanostructured electrodes, e.g. the evaporation of metals on electrospun fibres [5] and the deposition of metals on a thin film gel cracked by desiccation [18,19]. Metallic films with ordered nanoholes produced by nanosphere lithography showed good electrical conductivity, but low optical transparency [20-23]. Highly symmetric structures cause additional optical absorption due to the interaction of light with surface plasmons, which can, both, reduce the overall transmission, but also enhance it for specific wavelength bands (extraordinary optical transmission) [24].

Wire-grid electrodes can be optimised for onedirectional conductivity. A structure of aligned nanowires perpendicular to the side contacts leaves a higher portion of apertures for light transmission and reduces the symmetry. Transparent conductors with one-directional conductivity and excellent properties were produced by metal transfer from a PDMS stamp [6,25]. Even higher quality was achieved in a two-step process by deposition of copper mesoscale wires covered by silver nanowires (below $1 \Omega / \square$ sheet resistance and over $90 \%$ optical transmission) [26].

Nanosphere lithography (NSL) was already used for the production of anisotropic metallic grids, which requires monocrystalline and oriented nanospheres masks [27,28]. 
Here we present an alternative method based on the deposition at variable incidence angle, which benefits from a simpler geometry of the evaporation chamber. A metallic structure of thick parallel lines with thin interconnections was deposited, which lead to excellent sheet resistance below $3 \Omega / \square$ and good transparency with an easy production method. Due to the geometry, the optical transmission is higher for light polarised perpendicularly to the lines, which can be exploited for particular applications absorbing or emitting polarised light (e.g. liquid crystal displays). Depending on the application, apertures in the 100$1000 \mathrm{~nm}$ range can be achieved.

\section{Experimental methods}

Nanostructured films of gold or silver were produced by nanosphere lithography and physical vapour deposition on glass substrates.

2.1 Nanosphere Lithography Monolayers of polystyrene (PS) nanospheres with $754 \mathrm{~nm}$ diameter were deposited on a clean distilled water surface with a specially bent pipette [29]. For a $500 \mathrm{~cm}^{2}$ surface, $500 \mu \mathrm{L}$ of a $5 \%$ $\mathrm{w} / \mathrm{v}$ colloidal dispersion of nanospheres in water-ethanol mixture were used. The spheres spontaneously formed crystalline domains with hexagonal symmetry. Carefully inducing small waves on the surface helped rearranging and aligning the domains to form larger crystals (over 200 $\mathrm{cm}^{2}$ ). Introducing a tiny quantity of oil or grease on the water surface squeezed the spheres together until close packing was reached. The monolayer was deposited onto glass substrates by evaporation of the water (see Figure in the Abstract). The spheres were reduced to $2 / 3$ of their size by reactive ion etching in an oxygen-argon plasma according to Akinoglu et al. [30] in a MiniFlecto RIE chamber from Plasma Technology. The orientation of the crystal was determined by diffraction of a $532 \mathrm{~nm}$ laser beam. The laser wavelength should be about $60-90 \%$ of the periodicity (i.e. original size of the spheres); therefore if the periodicity is lower than $450 \mathrm{~nm}$ or higher than $1 \mu \mathrm{m}$, the orientation of the mask shall be determined by microscopy.

2.2 Physical vapour deposition Metallic films were deposited onto the glass substrates through the NSL masks. The deposition was performed at variable incident angle by tilting the sample, so that oval apertures with different aspect ratio could be achieved as shadows of the spheres: the short axis remained equal to the sphere diameter, the long axis increased for higher angles. The samples were mounted in the deposition chamber such that the spheres rows were aligned with the long axes of the ovals. There is a critical deposition angle at which the long axis reaches the periodicity, i.e. the oval apertures percolate [31]. The critical angle can be determined by microscopy (AFM or SEM) or by electrical conductivity measurements (see Section 3). For this work, $60-70 \mathrm{~nm}$ of silver or gold were deposited on a 1-2 nm thick layer of titanium for better adhesion on the glass substrate. The evaporation was performed at a distance of $15 \mathrm{~cm}$, a vacuum of
$10^{-7} \mathrm{hPa}$ and a deposition rate of $1 \mathrm{~nm} / \mathrm{min}$. The first part of the metal deposition (15-20 nm) was performed just below the critical angle $\left(40^{\circ}\right.$ to the perpendicular) and originated a film with oval apertures. The incidence angle was then increased $\left(60^{\circ}\right.$ to the perpendicular) to deposit thick lines parallel to the long axis of the oval apertures $(150 \mathrm{~nm}$ width and 45-50 nm thickness), as visible in Fig. 1. The lines are responsible for the biggest amount of the electrical conductivity. The underlying film with holes provides interconnections that bypass defects in the lines. After metal deposition, the spheres were removed by sonication in toluene. The samples were annealed for $3 \mathrm{~h}$ in vacuum at $300^{\circ} \mathrm{C}$ for gold nanostructures or $120^{\circ} \mathrm{C}$ for silver nanostructures.

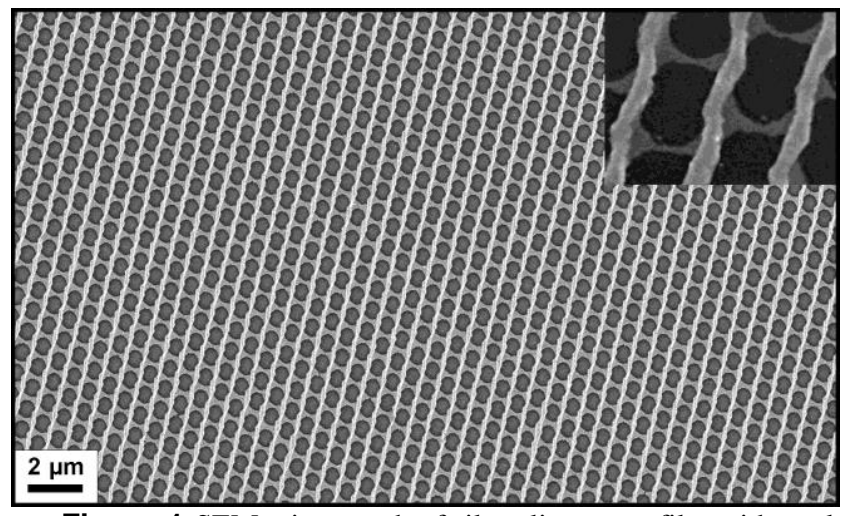

Figure 1 SEM micrograph of silver lines on a film with oval apertures.

2.3 Characterisation Optical transmission was measured between $300 \mathrm{~nm}$ and $1100 \mathrm{~nm}$ with a Thermo Scientific Evolution Array UV-Vis-IR spectrometer and the average value in the visible spectrum was evaluated. For the sheet-resistance determination, silver electrodes were deposited at the sides of the sample perpendicularly to the lines. Four-point measurements were performed with a current of $0.1 \mathrm{~A}$.

3 Results First, we analysed the properties of simple metallic films with oval apertures. A deposition at perpendicular angle $\left(0^{\circ}\right)$ produced a film with circular apertures, which had high conductivity but low transparency due to the small apertures and high symmetry of the structure (Fig. 2). Increasing the deposition angle elongates the apertures to ovals, which then percolated forming lines [31]. This reduced the symmetry and increased the aperture size for light transmission. As a consequence, we observed a strong increase in transparency with little losses in conductivity up to the critical angle at which the ovals percolate. In particular, we measured for a deposition angle of $40^{\circ}$ unchanged electrical conductivity in the long-axis direction and $70 \%$ higher transparency. For higher angles, the structure consisted of lines, whereby single defects or damages were sufficient to block the electrical conductivity of whole lines, with a sudden drop in the conductivity. 


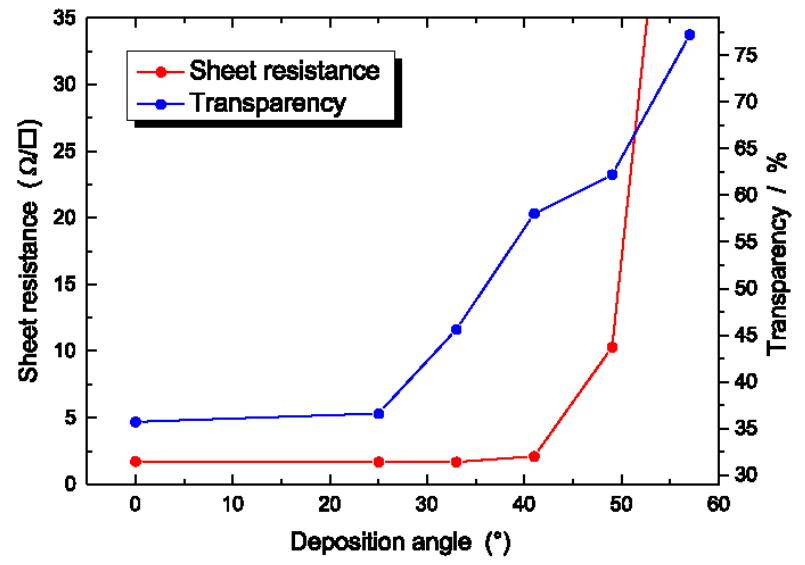

Figure 2 Sheet resistance and optical transparency of a $60 \mathrm{~nm}$ thick silver film with oval holes. A clear increase in resistance is measured at the deposition angle corresponding to the percolation of the oval holes, i.e. after breaking of the interconnections. Notice that these results show the sheet resistance of the film without thick lines for improved electrical conductivity.

The superposition of thick lines on the film with oval apertures (Fig. 1) allowed combining the excellent optical and electrical properties of the lines and the bypassing of defects by the thin interconnections. This lead to a sheet resistance along the lines below $10 \Omega / \square$ for silver films with $60 \%$ optical transmission and $5 \Omega / \square$ for gold films with $70 \%$ transmission. Perpendicularly to the lines a sheet resistance of over $1000 \Omega / \square$ was measured, which is in agreement with the results of Morfa et al for very thin conducting paths [32]. We noticed that lattice defects interrupting the lines (e.g. due to spheres of wrong size or crystal lattice defects) usually create additional interconnections in the vicinity. In this way, the line defects are efficiently bypassed in spite of the very high sheet resistance perpendicularly to the lines. Despite the higher cost, gold films also have a transmission spectrum better fitting the visible and NIR spectrum of the sun (Fig. 3), which is convenient for most applications. The silver nanostructures have an extraordinary optical transmission peak at $325 \mathrm{~nm}$, the gold nanostructures weaker peaks at $500 \mathrm{~nm}$ and 1100 $\mathrm{nm}$. The EOT peaks can be shifted by changing the periodicity of the nanostructure, i.e. the sphere size of the lithographic mask. They are more pronounced for higher symmetry (low deposition angle), whereby an increase in symmetry reduces the overall transmission due to plas-

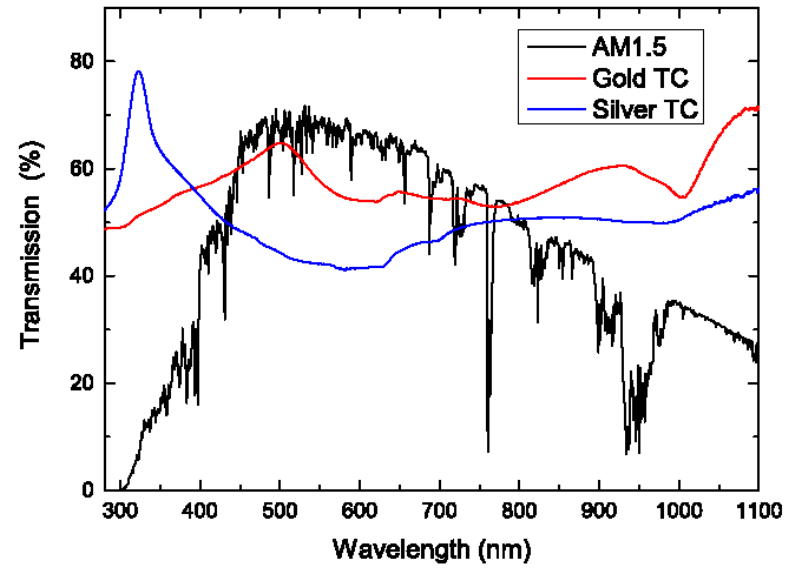

Figure 3 Transmission spectra of gold and silver films with oval apertures (deposition angle of $33^{\circ}$ ). While gold performs better in the optical and NIR range, silver has a very pronounced UV transmission peak. For comparison, the standard solar spectrum AM1.5 is shown.

4 Conclusion We presented a method for the production of metallic-grid transparent conductors with a mesh in the submicrometre range. They have very low sheet resistance and high transparency in the optical, UV-A and NIR range. For comparison, the well-established ITO electrodes have higher optical transmission (about $80 \%$ for 200-300 nm thickness) but usually higher sheet resistance of $10-100 \Omega / \square[33,34]$. The structure was formed by selfassembled nanosphere lithography masks without the need of advanced nanolithographic systems. A structure of thick, highly-conducting lines with thin interconnections was achieved with a single metal deposition at different incidence angles. Depending on the application, the material can be optimised for higher transmission or higher transparency by variation of line width and deposition angles. Moreover, the aperture size can be tuned using spheres of different size.

Acknowledgements VO acknowledges the German Academic Exchange Service (DAAD) for financial support. We thank Niclas S. Müller for the help with the construction of the PVD system, Eser M. Akinoglu and Anthony J. Morfa for sharing details about the NSL mask production.

\section{References}

[1] K. L. Chopra, S. Major, and D. K. Pandya, Thin Solid Films 102, 1 (1983).

[2] T. Minami, Thin Solid Films 516, 5822 (2008).

[3] R. B. H. Tahar, T. Ban, Y. Ohya, and Y. Takahashi, J. Appl. Phys. 83, 2631 (1998).

[4] A. C. Tolcin, 2011 Minerals Yearbook (U.S. Department of the Interior, U.S. Geological Survey, 2011).

[5] H. Wu, D. S. Kong, Z. C. Ruan, P. C. Hsu, S. Wang, Z. F. Yu, T. J. Carney, L. B. Hu, S. H. Fan, and Y. Cui, Nat. Nanotechnol. 8, 421 (2013). 
[6] M.-G. Kang and L. J. Guo, J. Vac. Sci. Technol. B 25, 2637 (2007).

[7] L. Ke, R. S. Kumar, P. Chen, L. Shen, S.-J. Chua, and A. P. Burden, Photonics Technol. Lett. IEEE 17, 543 (2005).

[8] W. Lee, R. P. Dwivedi, C. Hong, H. W. Kim, N. Cho, and C. Lee, J. Mater. Sci. 43, 1159 (2008).

[9] H. Kim, J. S. Horwitz, S. B. Qadri, and D. B. Chrisey, Thin Solid Films 420, 107 (2002).

[10] A. E. Rakhshani, Y. Makdisi, and H. A. Ramazaniyan, J. Appl. Phys. 83, 1049 (1998).

[11] M. Layani, A. Kamyshny, and S. Magdassi, Nanoscale 6, 5581 (2014)

[12] B. O'regan and M. Grfitzeli, Nature 353, 737 (1991).

[13] J. Van de Groep, P. Spinelli, and A. Polman, Nano Lett. 12, 3138 (2012).

[14] T. M. Barnes, M. O. Reese, J. D. Bergeson, B. A. Larsen, J. L. Blackburn, M. C. Beard, J. Bult, and J. Van de Lagemaat, Adv. Energy Mater. 2, 353 (2012).

[15] S. Günes, H. Neugebauer, and N. S. Sariciftci, Chem. Rev. 107, 1324 (2007).

[16] S. Hong, J. Bae, B. Koo, I. Chang, G. Y. Cho, Y.-B. Kim, S. W. Cha, and F. B. Prinz, Int. J. Precis. Eng. Manuf. Technol. 1, 67 (2014).

[17] R. B. Bergmann, Appl. Phys. A 69, 187 (1999).

[18] B. Han, K. Pei, Y. Huang, X. Zhang, Q. Rong, Q. Lin, Y. Guo, T. Sun, C. Guo, and D. Carnahan, Adv. Mater. 26, (2013).

[19] B. Han, Q. Peng, R. Li, Q. Rong, Y. Ding, E. M. Akinoglu, X. Wu, X. Wang, X. Lu, Q. Wang, G. Zhou, J.-M. Liu, Z. Ren, M. Giersig, A. Herczynski, K. Kempa, and J. Gao, Nat. Commun. 7, 12825 (2016).

[20] N. Kwon, K. Kim, S. Sung, I. Yi, and I. Chung, Nanotechnology 24, 235205 (2013).

[21] Y.-H. Ho, K.-Y. Chen, S.-W. Liu, Y.-T. Chang, D.-W Huang, and P.-K. Wei, Org. Electron. 12, 961 (2011).

[22] A. J. Morfa, E. M. Akinoglu, J. Subbiah, M. Giersig, and P. Mulvaney, J. Appl. Phys. 114, (2013).

[23] T. Gao, B. Wang, B. Ding, J. K. Lee, and P. W. Leu, Nano Lett. 14, 2105 (2014)

[24] T. W. Ebbesen, H. J. Lezec, H. F. Ghaemi, T. Thio, and P. A. Wolff, Nature 391, 667 (1998).

[25] M.-G. Kang, H. Joon Park, S. Hyun Ahn, and L. Jay Guo, Sol. Energy Mater. Sol. Cells 94, 1179 (2010).

[26] P.-C. Hsu, S. Wang, H. Wu, V. K. Narasimhan, D. S. Kong, H. R. Lee, and Y. Cui, Nat. Commun. 4, (2013).

[27] C. L. Haynes, A. D. McFarland, M. T. Smith, J. C. Hulteen, and R. P. Van Duyne, J. Phys. Chem. B 106, 1898 (2002).

[28] T. Qiu, E. M. Akinoglu, B. Luo, M. Giersig, M. Liang, J. Ning, and L. Zhi, Part. Part. Syst. Charact. (2016).

[29] P. Patoka and M. Giersig, J. Mater. Chem. 21, 16783 (2011).

[30] E. M. Akinoglu, A. J. Morfa, and M. Giersig, Langmuir (2014).

[31] A. J. Morfa, V. Oddone, and M. Giersig, Phys. Status Solidi (RRL)-Rapid Res. Lett. 10, 404 (2016).

[32] A. J. Morfa, E. M. Akinoglu, J. Subbiah, M. Giersig, and P. Mulvaney, J. Appl. Phys. 114, (2013).

[33] M. Mazur, D. Kaczmarek, J. Domaradzki, D. Wojcieszak, S. Song, and F. Placido, in Adv. Semicond Devices Microsystems (ASDAM), 2010 8th Int. Conf.
(2010), pp. 65-68.

[34] K. Ellmer, Nat. Photonics 6, 809 (2012). 